\title{
Constructing and evaluating a nursing capstone course for cultivating creativity in RN-BSN students in Taiwan
}

\author{
Ya-Lie Ku, Pei-Yu Lee, Man-Hua Shen, Chien-Lin Kuo \\ Department of Nursing, Fooyin University, Kaohsiung City, Taiwan.
}

Correspondence: Ya-Lie Ku. Address: No.151, Chin-Hsueh Rd., Ta-Liao District, Kaohsiung City 83102, Taiwan, R0C. Email: ns126@fy.edu.tw

Received: February 8, 2014

DOI : $10.5430 /$ jnep.v4n7p175
Accepted: March 28, 2014

URL: http://dx.doi.org/10.5430/jnep.v4n7p175
Online Published: June 15, 2014

\begin{abstract}
The purpose of this study was to construct and evaluate a nursing capstone course designed to cultivate creativity in RN-BSN students. The pre-post study design was used in a one-group of RN-BSN students who enrolled in a nursing capstone course. This pilot study was conducted under the auspices of NSC research grant 101-2511-S- 242-002, IRB protocol number FYH-IRB-101-07-01-A. The nursing capstone project course lasted for 18 weeks was taught for $2 \mathrm{hr}$ /week, and involved 6 mainstream methods, and 5 major assessment criteria. The 8 RN-BSN students' self-perceived abilities of creativity in this pilot study showed statistically significant improvement after completing the nursing capstone project course $(p<.01)$, but their characteristics, abilities, and motivation for creativity were unaffected. However, the measured barriers to creativity among 8 RN-BSN students decreased substantially from the pre-to the post test $(p<.05)$. Moreover, 9 RN-BSN students provided positive feedbacks about their learning experiences. Finally, the 9 RN-BSN students in the nursing capstone project course were developed two nursing products named as Anti-Needlestick Treasure Box and Comfortable Catheter Stickers, which have participated in the creative competition activities.
\end{abstract}

\section{Key words}

Creativity, Courses, Nursing students

\section{I ntroducation}

Because the Department of Education strongly encouraged creative education ${ }^{[1]}$, many technological colleges and universities in Taiwan have offered capstone creative courses that teach about innovative products to help students learn about applying for patents. Nursing professionals and medical institutions also urged nurses to play creative games and learn about innovative products and protocols to improve patients' comfort and satisfaction in the clinical settings ${ }^{[2,3]}$. The purpose of this study was to construct and evaluate a nursing capstone course designed to cultivate creativity in nursing students. The author constructed the nursing capstone project course with approval from the department of nursing at the institution where this study was constructed.

\section{Literature review}

This review of literature was divided into three sections that present creative teaching strategies, evaluation of creativity, and the effect of teaching creativity on students' creativity. In general education, Instructors used diverse teaching 
methods and strategies to cultivate students' creative thinking abilities ${ }^{[4]}$. Chen ${ }^{[5]}$ proposed a creative teaching model named Asking, Thinking, Doing, and Evaluating (ATDE), which involved asking probing questions about the ways of listing, comparing, substituting, imaging, and constituting (A); offering the thinking space and time (T); writing, speaking, drawing, performing, and discussing (D); and self-evaluating (E). Shen ${ }^{[6]}$ also introduced Creative Problem-Solving (CPS), which has three components, understanding problems, inspiring ideas, and preparing for action, and six steps: finding conflicts, searching for data, identifying problems, constructing ideas, answering, and searching for acceptable ideas.

In nursing education, $\mathrm{Ku}$, Sheu, and Kao ${ }^{[7]}$ proposed the concepts of fluency, flexibility, and uniqueness in nursing interventions, which were designed for students in a course on professional nursing concepts aimed at cultivating their creativity. Hsu, Chang, and Hsieh ${ }^{[8]}$ developed mind mapping as a visual thinking method to train nursing students in radiant thinking to cultivate their creative-thinking and problem-solving abilities. Furthermore, Huang and Hsu ${ }^{[9]}$ used mind-mapping and pithy rhymes for image learning in creative teaching to train nursing students quickly in intra-aortic balloon pump (IABP) standardized procedures. Chang and Hsu ${ }^{[10]}$ applied Mandala and mind-mapping to simplify the foot-examination process, and to enhance teachers' memory of the contents and procedures of foot examination by using the skills of mind-mapping and pithy rhyme.

For evaluating creativity, Taylor ${ }^{[11]}$ suggested indicators of creative thinking that were suitable for assessing nurses' learning outcomes regarding innovative nursing procedures or products. Story and Butts ${ }^{[12]}$ proposed that, in addition to helping evaluate learning outcomes, innovative teaching involved transforming the traditional learning process into teacher-students interaction and role-playing to cultivate nursing students' creativity; however, this required teachers to be curious and to have creative motivation to bring excitement into the teacher-student learning process. Based on a systematic literature review, Chan ${ }^{[13]}$ identified four learning patterns for creative thinking: diversity, freedom, confidence, and team-cooperation.

To investigate whether creative teaching was effective in enhancing students' creativity, Hsiao, Chang, and Huang ${ }^{[14]}$ examined the roles of fluency, flexibility, originality, elaboration and total creative-thinking abilities in figural and verbal scores, and in idea-design; although none of these factors were significantly different between the study's experimental group ( $(\mathrm{N}=18)$ and control group $(\mathrm{N}=8)$, the students and teachers who participated enjoyed cooperative learning and indicated that the approach could improve their creative abilities. Ku et al. ${ }^{[15]}$ applied ATDE in creative teaching strategies in a course on professional nursing concepts that addressed fluency, flexibility, and uniqueness and determined that fluency and flexibility, but not uniqueness, improved substantially over three semesters in students enrolled in a 2-years nursing program. Chao, Tai, \& Chiu ${ }^{[16]}$ studied how teaching strategies for creative thinking in an automobile practical course affected 42 students' creative temperament, and the researcher indicated that the course helped develop elaboration and headline description, but not facility and creative tendencies, and that those who had positive attitudes toward creative thinking teaching strategies convinced themselves to develop creative abilties.

Wang, Yu, and Wang ${ }^{[17]}$ explored the effectiveness of a design-based curriculum on the technological creativity of 76 junior high school students and found that the course enhanced conceptual, technical, and product-related creativity in student teams, and also improved students' abilities to design, create, be fluent, accommodate, and make progress. Chan, Huang, and $\mathrm{Wu}^{[18]}$ reported that fluency, flexibility, originality, and elaboration in figural creative-thinking abilities did not improve in their experimental-group students $(\mathrm{N}=87)$ who completed a creative-thinking course on home economics compared with students in the control group $(\mathrm{N}=68)$; by contrast, the verbal creative-thinking ability of the students in the experimental group was enhanced. Chao, Liu, Chang, and Chiu ${ }^{[19]}$ studied vocational high school students who completed a technological creative strategies course, and reported that students' creativity in terms of flexibility, originality, appearance, material knowledge, humaneness, manufacturing feasibility, and learning of technology were enhanced considerably in the experimental group ( $\mathrm{N}=37$ ) compared with the control group $(\mathrm{N}=38)$. Instead of a quantitative study, Dai, Chen, and Yuan ${ }^{[20]}$ conducted a qualitative case study on a vocational high school course involving storytelling about innovators that was designed to promote the technical creative-thinking abilities of students for problem-solving; based on 
analyzing data collected from interviews, the researchers reported that the course changed students' learning attitudes and behaviors, and increased students' competencies interest in acquiring professional knowledge, and in observing their surroundings carefully, and willingness to cultivate their own creativity.

This author applied creative-teaching strategies to a nursing capstone course by leading, discussing, reflecting, and brainstorming about CPS teaching methods ${ }^{[6]}$, and merged the ATDE teaching model ${ }^{[5]}$ with the concepts of fluency, flexibility, and uniqueness and with the skills of association, connection, substitution, and transformation. Innovative nursing products were evaluated using the five major criteria developed by the investigator following the creative evaluation guide from the National Union of Nurses' Association: importance, design process, literature review and rationale based, professional appropriateness, and value of application.

\section{Methods}

This was a pilot study conducted under the auspices of NSC research grant 101-2511-S-242-002, IRB protocol number as FYH-IRB-101-07-01-A. A nursing capstone course was developed as the 2 hours elective course for the students in an RN to BSN program without doing clinical practicum during spring semester. The investigator explained the purpose, process, concerns, and benefits of the study to each RN-BSN student who enrolled in the nursing capstone project course at the first week of the course. After consideration of participating in the course, they would obtain the signed informed-consent forms; however, they also could withdraw the course within two weeks.

The study merged creative strategies into the course of nursing capstone project course to cultivate the creativity in RNBSN students. The capstone project course was designed to guide nursing students in small teams to complete a project on a product related to practicum themes in the nursing profession. In addition to lecturing on the project production, the course introduced and discussed innovative protocols, interventions, strategies of activities, and creative products in domestic nursing practicum areas. The course also promoted the core competencies of the creativity and innovation, problem-solving, interpersonal communication, and in-group cooperation among the students by using multiple teaching strategies, such as the group interaction, individual reflection, and brainstorming, to produce the experiences of completing nursing projects, thus providing the basis for future evidence-based nursing research.

After completing the course, nursing students were able to (a) understand the process of developing creative products in the nursing capstone project process; (b) reflect on the factors that influenced the production process; (c) be inspired to take interest in and become motivated about the process of production; (d) experience the production process; (e) produce the innovative protocols, interventions, activities, or products in the nursing capstone project. The nursing capstone project course lasted for 18 weeks and was conducted for $2 \mathrm{hr} /$ week. The course consisted of six main stream methods for creative motivation. The CPS model was used to identify and solve clinical problems; the ATDE model was applied coupled with the concepts of fluency, flexibility, and uniqueness to train the students in using the abilities of association, connection, substitution, and transformation to think about problems and solutions. The course was designed to teach about and then develop innovative nursing products, and group discussions were conducted to evaluate these products. The instructors led discussion on metacognition in groups of RN-BSN students to help them reflect on their definition of creativity, barriers to creativity, and learning experiences in the nursing capstone project course. Table 1 presents the mainstream methods and strategies used in the nursing capstone project course.

After completing the nursing capstone project course, each innovative nursing product developed was assessed by following specific evaluating criteria. There are five major evaluating criteria were importance, designed process, literature review and rationale based, professional appropriateness, and value of application. Many evaluating items under each evaluating criterion were developed by the investigator based on the standards used by the National Union of Nurses' Association to assess creative nursing products. The scoring ranged from 1 to 5 from below 60 (1) to 90-100 (5) as shown in Table 2, which listed the evaluation criteria for innovative nursing products. 
Table 1. Mainstream Methods and Strategies used in the Nursing Capstone Project Course

\begin{tabular}{|c|c|}
\hline Mainstream methods & Strategies \\
\hline Motivation of capstone project & Play Sudoku to inspire students' creative interesting and motivation \\
\hline Clinical plight & \multirow{3}{*}{$\begin{array}{l}\text { Applied CPS three components (understanding problems, inspiring ideas, preparing } \\
\text { to action) and six stage process (identify plight, find information, problems, ideas, } \\
\text { solution, and accepted ideas }{ }^{[6]} \text {, led students consider the individual plight during } \\
\text { clinical practicum, search and read professional nursing literature }\end{array}$} \\
\hline Literature reading and analysis & \\
\hline Creative ideas/CPS & \\
\hline ATDE Model & \multirow{3}{*}{$\begin{array}{l}\text { Fluency is the total numbers of problem-solving strategies; flexibility is the total } \\
\text { numbers of categories for problem-solving strategies classifying by function, } \\
\text { component, or attribute; uniqueness is to apply the concepts of association, } \\
\text { connection, substitution, transformation to produce the innovative nursing products }\end{array}$} \\
\hline Fluency, flexibility, uniqueness & \\
\hline $\begin{array}{l}\text { Association, connection, substitution, } \\
\text { transformation }\end{array}$ & \\
\hline Initial designed map & \multirow{3}{*}{$\begin{array}{l}\text { Naming the innovative nursing products by brainstorming of group students } \\
\text { following their drawing designed map and process, searching the supported } \\
\text { rationale and principles and producing the innovative products }\end{array}$} \\
\hline Naming/design process & \\
\hline Producing real products & \\
\hline Discussion and evaluation & $\begin{array}{l}\text { Group discussion and evaluation following the criteria of importance/design } \\
\text { process/literature review and rationale based/professional appropriateness/value of } \\
\text { application }\end{array}$ \\
\hline Metacognition among groups & Proposed own creative definitions, barriers to creativity, and learning experiences \\
\hline
\end{tabular}

Table 2. The Evaluation Criteria for Innovative Nursing Products

\begin{tabular}{|c|c|c|c|c|}
\hline $1(60 \downarrow)$ & $2(60-69)$ & $3(70-79)$ & $4(80-89)$ & $5(90-100)$ \\
\hline \multicolumn{5}{|c|}{ Importance } \\
\hline \multicolumn{5}{|c|}{ Problem-solving } \\
\hline Individu & entered & & & \\
\hline
\end{tabular}

\author{
Designed Process ( Originality, Practice, Application) \\ No plagiarize \\ Convenience and simple \\ Concepts of association and connection \\ Concept of transformation and substitution \\ Comfort \\ Art
}

\title{
Literature review and rationale based
}

Accuracy

Integration

Evidences-levels

\section{Professional Appropriateness}

Research

Promotion

Experts review

Quality of care

ISO or patent proved

\section{Value of Application}

Publication, writing books, education

Economic and cost benefits

Assist and function

Total scores 
Before conducting the nursing capstone project course, all teachers attended a training workshop for a brief introduction to the course to ensure consistency in instruction and evaluation, and to enable them to write down ideas for the reflection part of the course. Table 3 presented the workshop day, place, time, and training content for the teachers in the nursing capstone project course.

Table 3. Workshop Day, Place, Time, and Training Content for the Teachers in the Nursing Capstone Project Course

\begin{tabular}{ll}
\hline Day/Place/Time & Training Content \\
\hline 2013.3.5. & 1. Explain the nursing capstone course \\
D306-2 meeting room & 2. Ways to inspire interesting for the nursing capstone project course \\
2:00-3:00 & 4. ATDE model with the concepts of fluency, flexibility, uniqueness \\
& 5. Concepts of association, connection, substitution, transformation \\
& $\begin{array}{l}\text { 1. Explain the process of producing innovative nursing protocols, interventions, or products and } \\
\text { evaluation criteria }\end{array}$ \\
2. Principals of instructing group of students & 3. Demonstrate one innovation nursing product and four faculty members evaluate it \\
& 4. Writing the reflection ideas
\end{tabular}

Four faculty members evaluated one innovative nursing product, Catch-Drain Lightweight Package. The evaluation of teachers A and B teachers correlated significantly $(r=.680, p<.01)$, as did those A and C $(r=.679, p<.01)$, and only theses three faculty members (A, B, C) showed consistent evaluation ( ICC .619, $p<.05$ ). Moreover, the nursing faculty members preferred the illustrations and inspiration of teaching sections and considered the ATDE model, which contains the concepts of fluency, flexibility, and uniqueness, and playing the game Sudoku with creative pictures and videos to be most helpful; however, the faculty members questioned the application of the concepts of association, connection, substitution, and transformation in innovative nursing products, and suggested rearranging the schedule of the nursing capstone course and adding more teaching activities during class hours. The "reflection" ideas that the four nursing faculty members offered were presented in Table 4.

Table 4. "Reflection" Ideas that the Four Nursing Faculty Members Offered

\begin{tabular}{ll}
\hline Questions & Reflection ideas \\
\hline What do you mostly & -Illustrating the examples of innovative products \\
prefer part during the & - Inspiring the interesting for the nursing capstone course and CPS methods \\
training? & -ATDE model merged the concepts of fluency, flexibility, uniqueness \\
Which contents are & -Motivation to apply the creative thinking into the other courses to active \\
most helpful for you? & students' thinking and reinforce the creative teaching \\
What are your & -Playing Sudoku with the creative pictures and videos to enrich the course \\
inspiration? & content, becoming more interesting \\
$\begin{array}{l}\text { Which parts of training do you still } \\
\text { feel confused about? }\end{array}$ & -Confusion about the concepts of association, connection, substitution, \\
& transformation \\
What is your suggestion for the & -Encouraging more teaching activities during the classroom and less \\
course? & homework assignments \\
& -rearranging time schedule for the nursing capstone project course to offer \\
& more time for students to think and produce their innovative nursing \\
\end{tabular}

In addition to preparing teachers for the nursing capstone project course, the RN-BSN students in the course evaluated innovative nursing products and their self-perceived creative abilities by using 1-to10- point Visual Analogue Scale (VAS) and the Chinese Nursing Practicum Creative Process Questionnaire (CNPCPQ), which contained 50 items, 10 each on demographics, characters involved in NPCP, abilities involved in NPCP, barriers to NPCP, and motivation involved in NPCP. The reliable and valid CNPCPQ was developed by Ku et al. ${ }^{[21]}$ under the auspices of the NSC research grant 101-2511-S-242-002 from August, 2012 to July, 2013. 


\section{Results}

The 9 RN-BSN students in this pilot test had a mean age of 22 years (range 21 - 23); all were female, single, and had work experience of 0-1.67 years; $37.5 \%$ were clinical nurses, $25 \%$ and $37.5 \%$ were $\mathrm{N}_{0}$ and $\mathrm{N}_{3}$ nurses; and none of the nurses had creative-training or creative-practicum experiences. However, only 8 RN-BSN students completed pre and post tests. After completing the nursing capstone project course, the self-perceived creativity of the 8 RN-BSN students improved significantly $(p<.01)$, but their characteristics, abilities, and motivation for creativity were not found to change. However, barriers to creativity decreased significantly from pre- to post-test in the 8 RN-BSN students $(p<.05)$. Table 5 presented the creativity of the $8 \mathrm{RN}-\mathrm{BSN}$ students after completing the course of nursing capstone project course.

Table 5. The Creativity of the 8 RN-BSN Students after Completing the Course of Nursing Capstone Project Course

\begin{tabular}{lllllll}
\hline items & mean & SD & SE & t & Df & sig \\
\hline Self-perceived the abilities of creativity & -2.500 & 1.927 & .681 & -3.669 & 7 & .008 \\
Characters & -3.875 & 5.768 & 2.039 & -1.900 & 7 & .099 \\
Abilities & -1.750 & 5.497 & 1.943 & -.900 & 7 & .398 \\
Barriers & 4.125 & 4.941 & 1.747 & 2.361 & 7 & .050 \\
Motivation & -2.000 & 3.024 & 1.069 & -1.871 & 7 & .104 \\
\hline
\end{tabular}

In addition to these quantitative evaluations, qualitative data were obtained from the reflection on the definitions of creativity and barriers to creativity in the 9 RN- BSN students; these data were presented in Table 6 . The 9 RN-BSN students defined creative mainly in terms of the problems-solving skills they could apply in the clinical settings to improve patient care by using the strategies taught by their instructors in the nursing capstone project course. The barriers to creativity among the 9 RN-BSN students included the limitations of time, cost, resources, and working experience, as well as their views on innovative nursing products, which agreed with the suggestions of the teachers.

Table 6. Reflection on the Definitions of Creativity and Barriers to Creativity in the 9 RN- BSN Students

\begin{tabular}{ll}
\hline Definition of Creativity & Barriers to Creativity
\end{tabular}

1) Clinical nurses identified the problems and plight, which associate with problem-solving by imagination during daily life.

2) Nursing creativity is for nurses to assure the security and improve the quality of patient care or environments.

3) Creativity is the unique idea, which could practice and apply into the nursing clinics, requiring the innovative and unique vision and ideas.

4) The ideas are coming from the connection and association with various products based on the nursing plight.

5) Creativity could alter the inconvenience in the clinical settings and reduce the working time for nurses

6) Applying creativity could change the current status by using nursing products.

7) Daily or clinical creative products could change inconvenient nursing working plight and make job work easily.

8) Completing the creative products is to play imagination, but produce practically.

9) Leading the creativity furthermore is to identify the plight and problems in the clinical settings using CPS and searching the reasons and reflecting how to alter and modify such plight or problems.

1) Time and numbers of students

2) Time and less working experiences

3) Time and money; suspicion about the appropriate and usefulness of the innovative nursing products

4) Sometimes flying ideas might go to the ending when encountered the real plight

5) I am the practical person and if I think it is impossible to produce such products, then I might give up

6) Some products are easy to think about, but it is difficult to produce it into the real one

7) Because my profession is in the nursing, it is difficult for me to play the role in the other areas such as physics.

8) Because each faculty has the different ideas about the products, we hope teachers could integrate their ideas before giving us revised suggestions.

9) The innovative nursing products take long time to modify or improve and this course is time-consuming 
The learning experience of the 9 RN-BSN students in the nursing capstone project course were illustrated by the narratives presented following this paragraph. The overall views of the students could summarize as follows: the course was difficult, but felt worthwhile despite the hardship after the innovative nursing products they had created; the 2-year BSN program course was unlike the courses in the associate-degree program; the course made the students realize the crucial role in developing products of cooperation among team members and also of teachers' instruction; the students felt a sense of achievement and also felt self-confidence, after completing the creative competition activities.

"At the beginning, I did not understand the foundation of this course. What are creative nursing products? However, I come to understand what the course was all about gradually, thanks to the teacher's instructions using creative photos and reports from the previous assignments. By helping each other on the issue of clinical plight and discussing the matter step by step, we produced the nursing product accurately and attended the competition. I cherish this opportunity because it helped me learn about quality of patient care.” (Student 1)

"In the course, I was taught concepts and ways of thinking unlike in the associate-degree program, and in the 2-year BSN program. I am happy that the new products we developed might help nurses improce the quality of patient care.” (Student 2)

“This challenging and unique course that I completed this semester is unlike previous courses I have taken. This course is focused on innovation and application and also taught us to think quickly. After completing this course, I understand how to create products of which development requires time and energy and communication within the team. Although our product was not the best one, we gained experience through the process of creating it." (Student 3)

"In this course, my teachers and I spent time and energy to produce the products and got a chance to compete. Although considerable effort was required for creating the products, we learned a lot, and for the first time after seven years of studying nursing, we understood the basic rationales and skills in nursing that we can apply in the future in the clinical settings.”(Student 4)

"Although the literature required was difficult to find, I am happy that through cooperation we were able to produce the products. Although we did not win the competition, at least we worked together to create the products, which makes the hard work worthwhile” (Student 5)

"After completing this course, I think that we can tackle the problems faced by nurses or patients in the clinical setting, which is a good thing. If enough support and abilities are available, creative ideas can be applied into solving the shortcomings in nursing, which would be wonderful.” (Student 6)

"We often do not think about creativity often during routine course because teachers lecture and we simply follow their way of thinking. However, in this course, we had to think constantly and occasionally we felt tired, but when we saw the products we created, we felt happily despite the hard work that was required during the production process.” (Student 7)

"We helped each other with the teachers' suggestions and instructions to complete this course and create the products successfully. By the way, we also attended the creative competition, which was a special experience, and my thanks to the teachers for offering such wonderful activities.” (Student 8)

"I have not taken a creative course before, but this course gave me a chance to apply my creativity and has made me realize that I also have the creative abilities. When we created the product, I felt a great sense of achievement and it 
improved my self-confidence, although the journey was hard. During the creative process of the production, I understood the importance of cooperation within the team, and from this course I have gained several valuable experiences.” (Student 9)

The 9 RN-BSN students in the nursing capstone project course were divided into two groups with instructing by two faculties and following were two nursing products named as Medical Toolbox and Comfortable Catheter Stickers (see Figure 1), which have participated in the creative competition activities.
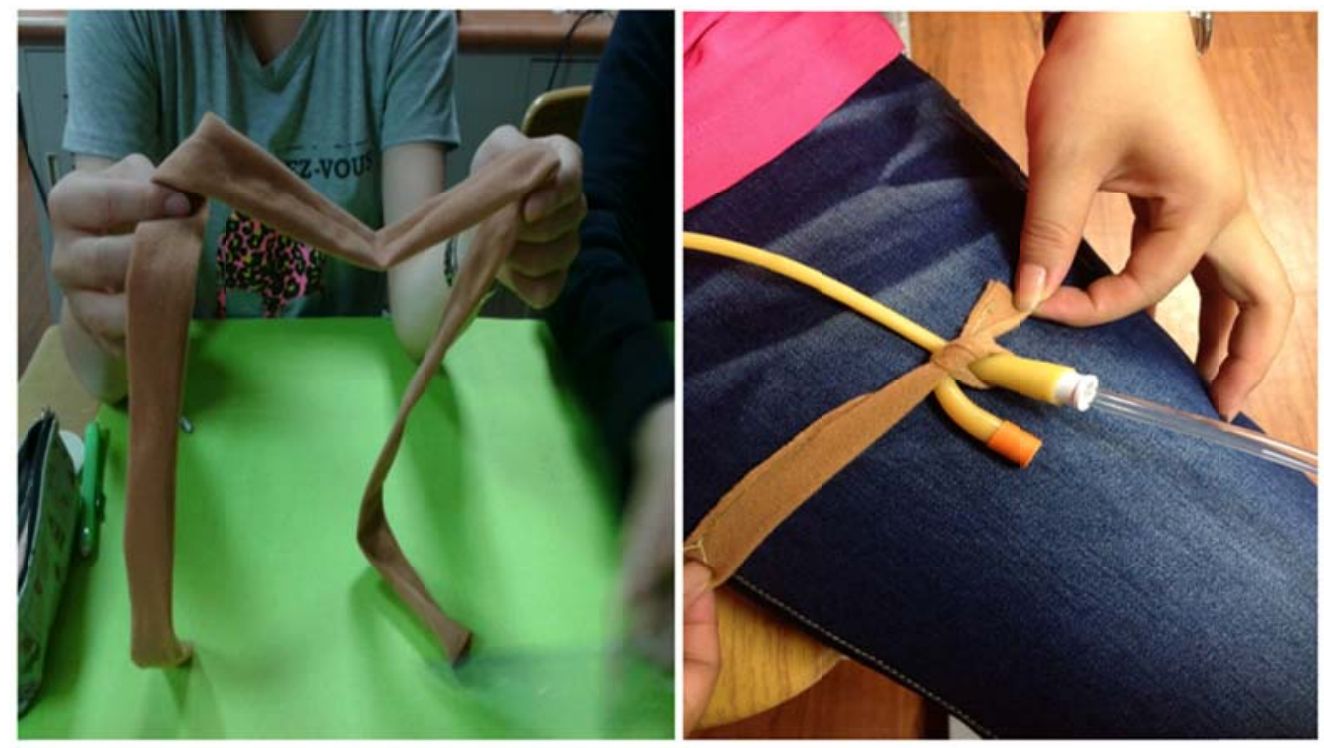

Figure 1. Comfortable Catheter Stickers

\section{Discussion}

Most of the literature on teaching creativity effectively to students was focused on students in high schools or vocational schools, and inconsistent findings have been reported because of different measurement tools being used or variations in sample sizes. Few studies were identified that were conducted on college or university students, especially nursing students. For creative teaching strategies, Hsu et al. ${ }^{[8]}$ used mind-mapping as the creative teaching strategy to cultivate nursing students' creativity. In 2011 and 2012 Hsu and coworkers combined mind-mapping with pithy rhymes to teach image learning, and used Mandala to teach clinical applications creatively. However, the author developed the classroom-based nursing course described here by applying the ATDE model ${ }^{[5]}$ and the CPS process ${ }^{[6]}$ coupled with the concepts of fluency, flexibility, and uniqueness and the skills of association, connection, substitution, transformation in the education areas that applied into the nursing classroom teaching. Additionally, evaluation of creativity has changed from using traditional instruments to assessing student-teacher interactions, subjective self-reports, and personal reflective journals or manuscripts records. Both traditional and humanistic evaluations were used in the study, including self-perception on creative abilities in the VAS, the CNPCP questionnaire, and reflection on definitions of creativity, barriers to creativity, and learning experiences.

\section{Conclusions}

In this pilot study, creativity in 8 RN-BSN students improved significantly regarding the students' self-perception of creative abilities after they completed the nursing capstone project course $(p<.01)$; but their characteristics, abilities, and 
motivation for creativity were found to be unaffected, which may be because of the small sample size. However, barriers to creativity decreased significantly from pre-to post-test in the 8 RN-BSN students $(p<.05)$. Furthermore, all 9 RN-BSN students indicated that their learning experiences were positive even though the course was challenging. This pilot study was an initial step toward developing a creative atmosphere in nursing education. The following recommendations were offered for future research in the areas. First, the experimental and control groups should be studied with larger sample sizes to validate the effectiveness of the nursing capstone project course in cultivating nursing students' creativity. Secondly, the innovative nursing products developed in the nursing capstone project course could be applied into the clinical settings to improve the quality of the patients' care. Third, a transcultural version of the CNPCP questionnaire and the nursing capstone project course could be used in nursing programs worldwide to increase the generalization and application of creative thinking skills in nursing education.

\section{References}

[1] Minister of Education. Taiwan Creative Education, 2004. Available from: http:// www. creativity .edu.tw

[2] Taiwan Nurse Association Message. Taiwan Nurse Association, 2005. Available from: http:// www.twna.org.tw

[3] Nursing Magazine. The National Union of Nurses’ Association, 2003\&2004. Available from: http:// www.nurse.org.tw

[4] Wu, C. S., \& Lin, T. U. Creative thinking teaching. Educational Information and Research. 2001; 39: 60-61.

[5] Chen, L. N. Creative Thinking Teaching Theory and Practice. Taipei: PSY, 2008.

[6] Shen, T. L. Creative Principle and Design. Taipei: Wu-Nan, 2008.

[7] Ku, Y. L., Sheu, S., \& Kao, C. H. The application of creative teaching strategies to the teaching protocol of nursing concepts. The Journal of Nursing. 2003; 50(3): 83-87.

[8] Hsu, L. L., Chang, M. Y., \& Hsieh, S. I. Mind mapping: A new tool for enhancing student learning strategy. The Journal of Nursing. 2008; 55(2): 76-80.

[9] Huang, Y. H., \& Hsu, L. L. Clinical teaching program: standardized operating procedure for intra-aortic balloon pump support. The Journal of Nursing. 2011; 59(4): 88-93.

[10] Chang, W. H., \& Hsu, L. L. A creative approach to teaching diabetic foot examinations. The Journal of Nursing. 2012; 59(6): 98-103.

[11] Taylor, B. N. Creativity fostering behaviors in the nurse educator (Unpublished doctoral Dissertation), University of San Diego, CA, USA; 2006.

[12] Story, L., \& Butts, J. B. Compelling teaching with the four Cs: Caring, comedy, creativity, and challenging. Journal of Nursing Education. 2010; 49(5): 291-294. PMid:20143753 http://dx.doi.org/10.3928/01484834-20100115-08

[13] Chan, Z. C. Y. A systematic review of creative thinking/creativity in nursing education. Nurse Education Today. 2012; 12(1): 21-27. PMid:21601528 http://dx.doi.org/10.1016/j.nepr.2011.04.008

[14] Hsiao, H. C., Chang, J. C., \& Huang, C. Y. The influence of cooperative learning on the creativity of undergraduate project work. Chinese Journal of Science Education. 2000; 8(4): 395-410.

[15] Ku, Y. L., Kao Lo, C. H., Wang, J. J., Lee Hsieh, J., \& Chen, K. M. The effectiveness of teaching strategies for creativity in a nursing concepts teaching protocol on the creative thinking of two-year RN-BSN students. The Journal of Nursing Research. 2002; 10(2): 105-112. PMid:12119595 http://dx.doi.org/10.1097/01.JNR.0000347589.98025.63

[16] Chao, C. Y., Tai, Y. K., \& Chiu, S. I. A study of the teaching effectiveness of automotive practical course with creative-thinking instructional strategy at the department of automobile of vocational high school. Journal of Technology Management. 2005; 10(1): 73-100.

[17] Wang, P. T., Yu, K. C., \& Wang, D. M. The study of effect of design-approach curriculum on student's technological creativity. Educational Journal of NHCUE. 2006; 22: 77-103

[18] Chan, C. H., Huang, H. H., \& Wu, M. H. A study of the teaching effectiveness of creative thinking in the home economics course. Journal of Technological and Vocational Education. 2009; 3(1): 43-74.

[19] Chao, C.Y., Liu, P. T., Chang, T. C., \& Chiu, S. I. The study of technology creativity teaching effectiveness for vocational high school: with project work as an example. Kaohsiung Normal University Journal. 2010; 29: 51-71. 
[20] Dai, C. Y., Chen, W. F., \& Yuan, Y. H. A case study on the impact of storytelling as a teaching model to infuse creativity into the professional electronics subjects of a vocational high school. Journal of Technological and Vocational Education. 2009; 3(2): 41-71.

[21] Ku, Y. L., Kuo, C. L., Tu, C. T., Shen, M. H., Lee, P. Y. Developing a Chinese Nursing Practicum Creative Process Questionnaire (CNPCPQ) in Taiwan. Poster presentation conducted at the conference of the Sigma Theta Tau 24th International Nursing Research Congress, Prague, Czech Republic, 2013. 